\title{
Research of the MOOC Study Behavior Influencing Factors
}

\author{
Fang Xu \\ Educational Institute, Lanzhou University, Lanzhou, Gansu, 730000, China
}

\begin{abstract}
The model of MOOC study behavior influencing factors is built based on TAM3 model, and empirical research is done. The result shows that the MOOC study intention has significantly positive correlation with MOOC study behavior; subjective norm, image, job relevance and output quality have significantly positive correlation with MOOC perceived usefulness and subjective norm has significantly positive correlation with image; Computer self-efficacy, perceptions of external control, perceived enjoyment and objective usability have significantly positive correlation with MOOC perceived ease of use. The output quality has the most positive correlation with MOOC perceived useful. In all the original variates, subjective norm has the most total effect to MOOC behavior intention. Finally some countermeasures is discussed.
\end{abstract}

Keywords: MOOC; influencing factors; TAM3; countermeasure

\section{Introduction}

Nowadays, MOOC (massive open online courses) is developing rapidly, but the MOOC study effect requires improving. According to related statistics, the course completion rate on Coursera platform is only $7 \%-9 \%$. The data Jordan (2013) investigate to major platforms shows that although some MOOC courses' passing rate can reach 40\%, most MOOC passing rate cannot reach 10\%. In 198 respondents, registered course number is 3.78 per person, but the amount of learners that finally complete the course and obtain a certificate is respectively 0.48 and 0.32 averagely, and average completion rate and average rate of acquiring certificates is $12.7 \%$ and $8.47 \%$ (Jiang ling al at.) It is necessary to study the MOOC study behavior influencing factors, so that we can take certain measures to improve the behavior of MOOC learner. There is little research about the research of the MOOC study behavior influencing factors domestic and overseas now. This study is based on TAM3 model (Technology Acceptance Model 3).

Davis built TAM model (Technology Acceptance Model) ${ }^{[4]}$ in 1986, and TAM2 was developed based on TAM in 2000. Venkatesh (2008) puts forward TAM3 model

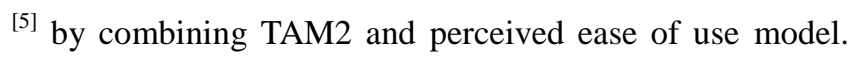
TAM3 model has many advantages such as it is comprehensive and has operable.

\section{Model building}

Based TAM3 model, combining MOOC practical situation, we build the MOOC influencing factors model (See Figure $1)$. 


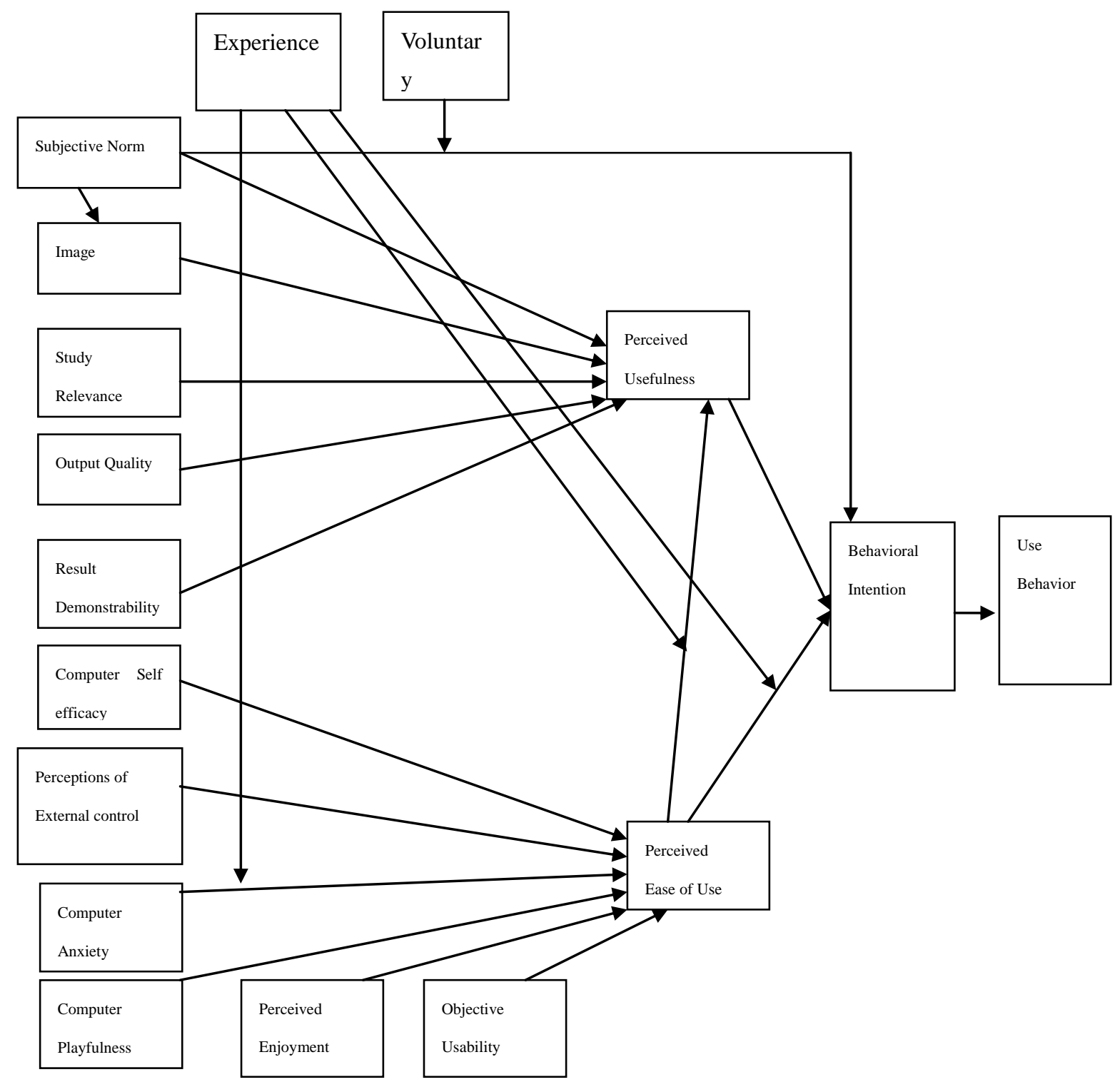

Figure 1 MOOC influencing model

In the model below, the influencing factor of use behavior is behavior intention; the influencing factors of behavior intention is perceived usefulness, perceived ease of use and subjective norm; The influencing factors of perceived usefulness are perceived ease of use, subjective norm, image, job relevance, output quality and result demonstrability; The influencing factors of perceived ease of use are computer self-efficacy, perception of external control, computer anxiety, computer playfulness, perceived enjoyment and objective usability.

\section{Empirical research progress and result}

We design questionnaire and issue questionnaires. Distributed form of the questionnaire includes network and paper distributing .Totally 325 effective questionnaires are recycled. There are $54.3 \%$ males and $45.7 \%$ females in the total effective questionnaires. There are $87.1 \%$ college students, and $12.9 \%$ are other people (including working people and so on). We use SPSS17.0 and AMOS 21.0 to 
deal with the data.

\subsection{Reliability and validity test}

We use Cronbach $\alpha$ Coefficient to verify the reliability of the data. The Cronbach $\alpha$ coefficient is computed for reliability test. The result is that Cronbach $\alpha$ coefficient of the variates are greater than 0.7 (except CANX=0.659), which shows the reliability of the data is good. The validity test includes convergent validity and cross validation. The AVE of all the variates is greater than 0.5 , and The CR of all the variates is greater than 0.7 , which shows the model has good convergent validity. The square root of AVE of all the variates is than the correlation coefficient between the variables, which shows that the data has good cross validation.

\subsection{Path graph analysis}

The path regression coefficient can be seen in table 1 .

Table 1 Regression coefficient $\left(\mathrm{P}^{* * *}<0.001\right)$

\begin{tabular}{|lll|ll|}
\hline & & & Estimate & P \\
\hline PEOU & $<---$ & CSE & .191 & $* * *$ \\
PEOU & $<---$ & ENJ & .183 & $* * *$ \\
PEOU & $<---$ & PEC & .094 & .077 \\
PEOU & $<---$ & CANX & -.061 & .206 \\
PEOU & $<---$ & OU & .164 & .001 \\
PEOU & $<---$ & CPLAY & .077 & .137 \\
IMG & $<---$ & SN & .383 & $* * *$ \\
PU & $<---$ & SN & .220 & $* * *$ \\
PU & $<---$ & PEOU & .007 & .911 \\
PU & $<---$ & REL & .160 & .004 \\
PU & $<---$ & IMG & .129 & .015 \\
PU & $<---$ & OUT & .413 & $* * *$ \\
PU & $<---$ & RES & .028 & .583 \\
BI & $<---$ & PU & .444 & $* * *$ \\
BI & $<---$ & PEOU & .116 & .065 \\
BI & $<---$ & SN & .317 & $* * *$ \\
USE & $<---$ & BI & .424 & $* * *$ \\
\hline
\end{tabular}

From the table 1, we can get the following conclusions $(\mathrm{p}<0.05)$ :

(1) The MOOC behavior intention has significant positive correlation with MOOC use. This conclusion is consistent with other research that TMA applies on other technology system.

(2) Subjective norm and perceived usefulness have significant positive correlation with MOOC behavior intention, this conclusion is consistent with other research that TMA applies on other technology system.

Perceived ease of use has positive correlation with MOOC behavior intention, but not significant. This conclusion is consistent with some other research that TMA application on other technology system, but not consistent with some research.

The perceived usefulness has the most positive correlation with MOOC behavior intention (regression coefficient is 0.444), and the following is 
subjective norm and perceived ease of use.

(3) Subjective norm, image, study relevance and output quality have significant positive correlation with perceived usefulness.

If the learner feels that the people who he thought is important to him think that he should study MOOC, the perceived usefulness of MOOC of the learner will improve significantly. Because if he studies MOOC, this will improve his status in the leader's mind. If he doesn't study MOOC, this will be probably influence his work. . When the learner feels MOOC can apply his work more, he will feel MOOC useful more significantly. If the learner feels MOOC can make his work better, he will feel MOOC more useful significantly.

Result demonstrability and perceived ease of use have significant positive correlation with perceived usefulness, but not significant.

Output quality have the most positive correlation with perceived usefulness(regression coefficient is 0.413)and the following is subjective norm, study relevance, image, result demonstrability and perceived ease of use in turn depending on the regression coefficient size.

(4) Computer self-efficacy, perceptions of external control, perceived enjoyment and objective usability have significant positive correlation with perceived ease of use. If the learner of MOOC has higher computer self-efficacy, he will have more confident to finish MOOC study and this will improve his perceived ease of use significantly. When the learner feels when he studies MOOC, he will deprive more help; his perceived ease of use will be improved significantly. When the learner feels more enjoyment studying MOOC, he will feel more perceived ease of use of MOOC study significantly .When the learner feels MOOC study has more advantages on operating, he will feel more perceived ease of use of MOOC study significantly.

Computer anxiety has negative correlation with perceived ease of use, but not significant. Computer playfulness has positive correlation with perceived ease of use, but not significant.

$\begin{array}{cccc}\text { Computer self-efficacy } & \text { have the most } \\ \text { positive correlation } & \text { with perceived ease of }\end{array}$

use(regression coefficient is 0.191) ,the following is perceived enjoyment, objective usability perceptions of external control and computer playfulness in turn depending on the regression coefficient size .

(5) Subjective norm has significant positive correlation with image. If the learner feels the leader of him hopes he studies MOOC, he will think that his status will improved if he study MOOC, because the leader plays an important role in his promotion or other appraising and so on.

We can see that subjective norm has the most effect to the behavior intention in all the original variates (total effect is 0.437). This gives us enlightenment when we make related policies.

\section{Countermeasure research}

\subsection{Vigorously propagandizing MOOC}

According to the investigation of writer, there are many people (college students) who have not known MOOC yet. We should strengthen propaganda of MOOC. Tsinghua university school online is famous in our country's MOOC. For example, Tsinghua university school online can send people to the university to propagandize their MOOC through lectures and so on. The foreign MOOC, such as edx, coursera and udacity, can go to other countries to propagandize their MOOC. They should introduce origin of MOOC, the change MOOC will bring the people, courses condition on the platform and so on. And this will enhance output quality, study relevance, perceived enjoyment and so on.

\subsection{Encouraging participation of MOOC}

The teachers and related people should encourage learner to attempt to study MOOC. As studying MOOC more and more, the learners may find more and more usefulness and ease of use of MOOC, which can promote their study of MOOC. Encouraging participation of MOOC may be a useful way to guide learners to study MOOC. We can encourage students to watch MOOC video, participate in interactive activities and so on. The teachers can combine MOOC courses with their teaching courses, for example, the teacher can use flipped classroom model to teach, where MOOC is used. 


\subsection{Management measures}

We can take some essential management measures to improve MOOC study. Taking management measures can improve subjective

norm, image, study relevance, result demonstrability, output quality and perceptions of external control. We should attempt to establish credit transfer system. Now the MOOC evaluation system is not consummate. Now the final test question topic is mainly objective questions, but the subjective question is less. The subjective test question should be increased. The related incentive countermeasures should be implemented. MOOC investigation report in China in 2014 of Shells net show that scholarship or other reward is one of the five big power of the MOOC future learning, which was chosen by $50.7 \%$ of respondents, ranking the third. MOOC can provide certain rewards to excellent students or other moral encouragement.

\section{Conclusion}

The influencing factors of MOOC study are researched in the paper through empirical research. The influencing factor model is built in the paper based on TAM3 model. The questionnaire is sent and the data is dealt with by SPSS17.0 and AMOS 21.0. The related conclusion is obtained, such as perceived usefulness has significant positive correlation with MOOC behavior intention. Finally some advice is put forward. We can use interview way for further research in future. We can discuss the influencing mechanism more deeply later. We should promote the development of MOOC vigorously.

\section{Reference}

[1] Jiang lin, Han xibing, Cheng jiangang, "MOOCs learner characteristics and Learning effect analysis and research", China Educational Technology, 2013(11): 54-65.

[2] http://en.wikipedia.org/wiki/MOOCs, 2013-08-03.

[3] Davis F D, “A Technology Acceptance Model of Empirically Testing New End - User Information Systems: Theory and Results". Sloan School of Management, Massachusetts Institute of Technology. 1986.

[4] Viswanath Venkatesh, "Technology Acceptance Model 3 and a Research Agenda on Interventions", Decision Sciences [J], 2008,39 (2): 273-315.

[5] Hao dan, "Document analysis of moocs research condition Domestic". Distance Education in China, 2013(11):42-50. 